\title{
25 Research Square

\section{Multi-center randomized trial of Compound Kushen injection for decreasing radiation- induced thoracic toxicity in lung cancer patients}

\section{Qingxi Yu}

Shandong Tumor Hospital

\section{Jun Wang}

Hebei Medical University Fourth Affiliated Hospital and Hebei Provincial Tumor Hospital

\section{Fan Wang}

The First Affiliated Hospital of anhui medical university

Haitao Yin

Xuzhou Center Hospital

\section{Xia Li}

Liaoning Provincial Tumor Hospital

\section{Shengli Yuan}

Qingdao Municipal Hospital

\section{Fujun Yang}

Weihai Municipal Hospital

\section{Bing Xia}

Hangzhou Cancer Hospital

\section{Zhongyao Jia}

Linyi People Hospital

Sen Liu

Jinan Central Hospital Affiliated to Shandong University

Kaixian Zhang

Tengzhou Central People's Hospital

\section{Xuechao Jiang}

Binzhou Central Hospital

Fengchun Liu

Siping First People's Hospital

\section{Luanqiu Wang}

Qingdao Central Hospital

\section{Junji Deng}

Weifang Traditional Chinese Medicine Hospital

Xu Tong 
Qiqihaer Tumor Hospital

\section{Shuyan Liu}

Harbin Chest Hospital

\section{Hongsheng Lin}

China Academy of Traditional Chinese Medicine Guanganmen Hospital

\section{Shuanghu Yuan ( $\square$ yuanshuanghu@sina.com )}

Shandong University, Shandong First Medical University and Shandong Academy of Medical Sciences, Shandong Cancer Hospital and Institute

\section{Research article}

Keywords: Compound Kushen Injection, non-small cell lung cancer, chemoradiotherapy, quality of life, radiation-induced thoracic toxicity

Posted Date: April 8th, 2021

DOI: https://doi.org/10.21203/rs.3.rs-135592/v2

License: (c) (7) This work is licensed under a Creative Commons Attribution 4.0 International License. Read Full License 


\section{Abstract}

Background: Thoracic radiation therapy plays an important role in the treatment of inoperable lung cancer. However, radiotherapy-induced toxicity poses an important challenge for radiation oncologists. Research has shown that Compound Kushen Injection (CKI) has anti-inflammatory activity and can reduce toxicity when given with chemotherapy. So, we hypothesis CKI can decrease chemoradiotherapyinduced thoracic toxicity in lung cancer patients and conducted this randomized, multi-center study.

Methods: A prospective, open, randomized, multi-center囚phase IV trial was performed, in which a total of 296 lung cancer patients were enrolled and randomly divided 1:1 into experimental and control groups. The control group received standard chemoradiotherapy including precise thoracic radiotherapy (60 Gy/30 fractions/ 6 weeks) plus concurrent platinum-based chemotherapy. The experimental group received standard chemoradiotherapy plus CKI via mainline treatments of $250 \mathrm{ml} \mathrm{CKI}(20 \mathrm{ml}$ diluted in $0.9 \%$ normal saline) daily for 20 continuous days. The incidence of adverse events and severity of thoracic toxicity after treatment were observed according to version 4.0 of the Common Terminology Criteria for Adverse Events. Adverse drug reactions (ADRs) and quality of life (QLQ-C30) also were compared between the two groups.

Results: Two hundred ninety-one qualifying patients were included in the statistical analysis. Symptomatic radiation-induced thoracic toxicity was lower in the experimental group than in the control group (14.1\% vs $25.6 \%$, $p=0.017$ ). According to QLQ-C30 questionnaire findings, CKI provided superior outcomes related to pain relief, relieving fatigue, social functioning and emotional functioning $(p<0.05)$. The rates of adverse events $(26.4 \%$ vs $30.8 \%, p>0.05)$ and severe adverse events $(5.4 \%$ vs $2.8 \%, p>0.05)$ did not differ significantly between the experimental and control groups.

Conclusion: CKI significantly alleviated symptomatic radiation-induced thoracic toxicity, as well as pain, when given with chemoradiotherapy for lung cancer treatment.

\section{Background}

Lung cancer is the leading cause of cancer-related mortality worldwide [1]. Moreover, most cases are stages III and IV, which are inoperable, at the time of diagnosis. For these patients, the standard treatment is radiotherapy with systematic platinum-based doublet chemotherapy. Unfortunately, severe side effects such as radiation-induced pneumonitis and esophagitis, fatigue, pain, vomiting, and drowsiness can seriously affect patients' quality of life (QoL) as well as the curative effects of treatment. Thus, reducing such toxicity and enhancing the curative effect of chemoradiotherapy remains a major challenge in lung cancer treatment.

Various traditional Chinese medicinal herbs have been used to reduce toxicity and enhance the curative effect of chemoradiotherapy in many types of cancers. Therapy integrating traditional Chinese and Western medicine has been the most widely used method for treating malignant tumors in China [2-5]. One compound in traditional Chinese medicine, Compound Kushen Injection (CKI) is extracted from the 
Kushen (Radix sophorae flavescentis) and Baituling (Rhizoma smilacis glabrae) herbs, and its primary components are oxymatrine and matrine $[6,7]$. Previous studies $[8,9]$ have indicated that CKI can reduce radiation-induced toxicity. The State Food and Drug Administration of China approved CKI for treating cancer in 1992 [10]. Since then, CKI has been extensively used in the Chinese clinical setting. Many clinical reports have demonstrated its anti-cancer effects against gastric cancer, liver cancer, and breast cancer [11-14]. It was reported that CKI attenuates the side effects of chemotherapy and radiotherapy by improving QoL, regulating immune function, and synergizing the therapeutic effects of chemotherapy and radiotherapy $[15,16]$. Furthermore, Yanju et al. [17] found that CKI had a positive effect on alleviating the pain of bone cancer; compared with radiotherapy or bisphosphonates alone, the addition of CKI showed positive improved pain relief and the Karnofsky Performance Status (KPS) in patients with bone cancer. The patients treated with CKI also achieved significant reductions in the incidence of leukopenia and nausea, and no patients in the CKI treatment group had to stop treatment due to adverse events.

According to the observed benefits of CKI in cancer patients, we hypothesized that CKI can reduce chemoradiotherapy-induced thoracic toxicity in lung cancer patients. The presented multi-center randomized trial tested this hypothesis by comparing the incidence of adverse events, the severity of thoracic toxicity, and QoL in lung cancer patients who received standard chemoradiotherapy with or without concurrent CKI.

\section{Methods}

\section{Patient recruitment}

This was a multicenter, randomized, open-labelled phase IV trial conducted in 20 hospitals. Recruitment began in October 2014 and ended in May 2016. Written informed consent was obtained from all patients before participation in the trial. A total of 296 lung cancer patients were randomly divided into the experimental group $(n=148)$ and the control group $(n=148)$, using sealed envelopes. Patients in both groups received standard treatment with radiotherapy plus platinum-based chemotherapy. The ethics committee of Guang'anmen hospital and Shandong cancer hospital approved this study and it met international standards or patient confidentiality. Written informed consent was obtained from each patient.

\section{Eligibility criteria}

Patients were eligible for inclusion in the study if they met all of the following inclusion criteria and had none of the listed exclusion criteria.

\section{Inclusion criteria:}

(1) before the start of the study, the patients fully understood the research and signed the informed consent form;

(2) age $18-75$ years; 
(3) pathologically proven lung cancer diagnosis;

(4) according to RECIST (version 1.1), at least one objectively measurable tumor lesion (iconography: computed tomography [CT], magnetic resonance imaging [MRI]) with a diameter $>10 \mathrm{~mm}$ (and malignant lymph nodes on CT scans had a diameter $>15 \mathrm{~mm}$ );

(5) lung function FEV1 more than at least $1 \mathrm{~L}$ and more than $50 \%$ A normal value;

(6) basically normal organ function: absolute neutrophil count (ANC) $>1.5 \times 10^{9} / \mathrm{L}$, platelet (PLT) count > $100 \times 10^{9} / \mathrm{L}$, hemoglobin $(\mathrm{Hb})>9.0 \mathrm{~g} / \mathrm{dl}$, total bilirubin (TBIL) level either normal or $<1.5 \times$ the upper limit of normal (ULN), aspartate transaminase (AST [SGOT]) and alanine transaminase (ALT [SGPT]) levels $<2.5 \times$ ULN ( if with liver metastases, $<5 \times \mathrm{ULN}$ ), and serum creatinine $(\mathrm{SCr})<1.5 \times \mathrm{ULN}$;

(7) expected survival time $>6$ months.

Exclusion criteria:

(1) any prior radiotherapy to the lung or mediastinum;

(2) treatment using CKI within 2 weeks before chemoradiotherapy ;

(3) pregnancy or lactation;

(4) severe, uncontrolled organ dysfunction or infection, such as decompensated heart, lung, or kidney failure, which can lead to intolerance of chemotherapy;

(5) participation, current or within the previous 30 days, in other clinical trials;

(6) hypersensitivity to any component of the trial regimen;

(7) history of serious psychological or psychiatric disorders, drug addiction, or alcohol dependence;

(8) and condition making sufficient compliance with this clinical trial unlikely.

\section{Study aims}

The primary aim of this study was to observe the incidence and severity of thoracic toxicity during chemoradiotherapy with and without CKI treatment. The secondary aims of this study were to compare clinical symptoms, QoL and adverse drug reactions (ADRs) between the experimental and control groups.

\section{Intervention}

Patients in the experimental group received mainline treatments of $250 \mathrm{ml} \mathrm{CKI} \mathrm{(} 20 \mathrm{ml}$ diluted in $0.9 \%$ normal saline, Shanxi Zhendong Pharmaceutical Co., Ltd., Shanxi, China) daily for 20 continuous days in combination with radiotherapy and chemotherapy. Patients in the control group received only 
radiotherapy and chemotherapy according to standard guidelines. The follow-up period extended to 4 months after the completion of radiotherapy.

\section{QoL assessment}

The European Organization for the Research and Treatment of Cancer Quality of Life (EORTC QLQ-C30) [18], which includes 30 items, was used to evaluate the impact of drugs on patients. According to patients' answers, the items were scored from 1-4 or 1-7 points. The EORTC QLQ-C30 items are divided into 15 domains, including 5 functional domains, 3 symptomatic domains, 1 life-quality domain, and 6 single items (each one acts as a domain). The raw score (RS) for every domain is the average score of all items in the domain, according to the following formula: $\mathrm{RS}=\left(\mathrm{Q} 1+\mathrm{Q} 2+{ }_{n, \ldots}+\mathrm{Qn}\right) / \mathrm{n}$. To compare the scores in every field with each other, we used the range method of linear transformation to transform the RSs into a standard score (SS), which ranged from 0-100. The following formulae for two areas were used (R stands for the score range): functional domain, $S S=[1-(R S-1) / R] \times 100$; and symptomatic and life-quality domains, $S S=[(\mathrm{RS}-1) / \mathrm{R}] \times 100$.

\section{Safety assessment}

Version 4.0 of the Common Terminology Criteria for Adverse Events (CTCAE) [19] issued by the National Cancer Institute $(\mathrm{NCl})$ was used for safety assessment. Liver and kidney function, routine blood analysis, urine, and stool testing, and electrocardiography were completed to assess drug toxicity and adverse reactions in the two groups.

\section{Ethics}

This trial was reviewed and approved by the XXXHospital affiliated XXX (approval number: XXX). The trial has been registered in the Chinese Clinical Trial Registry (registration number: XXX).

\section{Statistical analysis}

We used SPSS version 20.0 (SPSS, Inc., Chicago, IL, USA) for the statistical analyses. All continuous data are presented as the mean \pm standard deviation. Data for the groups were compared using independent sample t-tests. Differences in patient characteristics between the two groups were examined using the $t$ test. Values of $p<0.05$ were considered significant.

\section{Results}

The clinicopathological characteristics showed no differences between patients in the treatment and control groups (all $p>0.05$, Table 1 ).

Our comparison(Table 2) of the severity of radiation-induced thoracic toxicity between the two groups revealed that patients who received CKI treatment along with standard chemoradiotherapy experienced significantly less severe adverse events $(p=0.017)$.Specifically, the incidence and severity of radiationinduced pneumonitis was significantly lower in the experimental group than in the control group $(p=$ 0.001). However, no significant differences were observed between the groups in relation to the 
occurrence of radiation-induced esophagitis and radiation-induced heart damage (both $\mathrm{p} \varangle 0.05$ ), possibly because these forms of thoracic toxicity were rare in our study.

Overall, no significant differences in the incidence rates of adverse events and severe adverse events were observed between the experimental and control groups (both $p>0.05$; Table 3 ).

Upon assessment of QoL in the two groups (Tables 4), significant differences were found in the relief of pain and fatigue between the two groups (both $p<0.01$ ). Additionally, the experimental group scored higher for social function and emotional function (both $p<0.05$ ), further indicating that CKI provided superior QoL following chemoradiotherapy for lung cancer.

\section{Discussion}

According to traditional Chinese Medicine theory, eliminating pathogenic factors and strengthening genuine Qi are of equal importance in treating tumors: eliminating pathogenic factors includes killing tumor cells via radiotherapy or chemotherapy, and strengthening genuine Qi protects the immunologic functions of the organism via the use of drugs, to increase the immunity of the organism $[20,21,22,23]$. $\mathrm{CKI}$, known as the Yanshu injection, is extracted from two Chinese herbs, kushen (Radix sophorae flavescentis) and baituling (Rhizoma smilacis glabrae), for which the primary components are oxymatrine and matrine, respectively [24]. Clinically, positive benefits of CKI in the treatment of lung cancer have also been demonstrated [25]. In the treatment of elderly patients with advanced non-small cell lung cancer, CKI has been widely used because of its high efficiency and low toxicity [26].

In the past few decades, radiation techniques have developed rapidly, including three-dimensional conformal radiation therapy (3D-CRT), Intensity-modulated radiation therapy (IMRT), image-guided radiation therapy (IGRT), stereotactic body radiation therapy (SBRT), and proton and particle beam therapy [27]. However, radiation-induced pneumonitis still cannot be completely prevented, which restricts the therapeutic dose and effectiveness of treatment. There have been few studies on the use of CKI in patients receiving radiotherapy. In our study, the morbidity of radiation-induced pneumonitis was significantly less in the experimental group than in the control group. Moreover, the incidence rates of fatigue and pain were lower in the experimental group than in the control group. The social function and emotional function in the experimental group also were better than those in the control group. Consistent with our findings, Qin et al. [28] studied 86 elderly NSCLC patients who were randomly divided into an experimental group $(n=43)$ and a control group $(n=43)$, who received the docetaxel with carboplatin chemotherapy alone. The incidence rates of adverse reactions, including nausea and fatigue were lower in the experimental group than in the control group $(p<0.05)$. Lu Let al. [29] performed a systematic review on CKI adjuvant chemotherapy in the treatment of lung cancer that included 23 randomized controlled trials (1,750 cases) and found that CKI adjuvant chemotherapy was associated with a better objective response rate (odds ratio $[\mathrm{OR}]=1.68,95 \%$ confidence interval $[\mathrm{Cl}]=1.32-2.04, \mathrm{p}<0.0001$ ) and better $\mathrm{QoL}(\mathrm{OR}=2.69,95 \% \mathrm{Cl}=1.97-3.68, \mathrm{p}<0.00001)$ then chemotherapy alone. 
The present multi-center, randomized, controlled, open-label study of CKI in combination with radiotherapy in patients with locally advanced NSCLC supports and provides parameters for the use of CKI in combination with radiotherapy as a novel treatment approach for advanced NSCLC to reduce symptomatic radiation-induced thoracic toxicity.

We acknowledge that there are some limitations to the current study. First, patients of all ages were enrolled, which resulted in a heterogeneous distribution of adjuvant treatment procedures. Additionally, the duration of follow-up was short.

\section{Conclusions}

In summary, the addition of CKI administration to radiotherapy and chemotherapy regimens for lung cancer treatment appears to be able to reduce toxicity, which may improve patients' immunologic function and QoL. In Chinese clinics, we commonly administer CKI to synergize the therapeutic effects of radiotherapy and chemotherapy. Whether $\mathrm{CKI}$ can prolong the survival of patients with lung cancer requires further investigation.

\section{List Of Abbreviations}

Compound Kushen Injection (CKI), Adverse drug reactions (ADRs), quality of life (QLQ-C30) quality of life (QoL), Karnofsky Performance Status (KPS), Response Evaluation Criteria In Solid Tumors(RECIST), computed tomography [CT], absolute neutrophil count (ANC)

platelet (PLT), hemoglobin (Hb), total bilirubin (TBIL), upper limit of normal (ULN),

aspartate transaminase (AST [SGOT]), alanine transaminase (ALT [SGPT])

serum creatinine ( $\mathrm{SCr}$ ), The European Organization for the Research and Treatment of Cancer Quality of Life (EORTC QLQ-C30), raw score (RS), standard score (SS), the Common Terminology Criteria for Adverse Events (CTCAE), National Cancer Institute (NCl), three-dimensional conformal radiation therapy (3D-CRT), Intensity-modulated radiation therapy (IMRT),

image-guided radiation therapy (IGRT), stereotactic body radiation therapy (SBRT),

\section{Declarations}

Ethics approval and consent to participate,

The ethics committee of Guang'anmen hospital and Shandong cancer hospital approved this study and it met international standards or patient confidentiality. 
Written informed consent was obtained from each patient.

Consent for publication

All authors approved of the final version of the article, including the authorship list.

Availability of data and material

Not applicable

Competing Interests

The authors declare that they have no competing interests.

Funding,

Not applicable

Author contributions

HS L, SH Y conceived of and designed the experiments; QX Y, J W, F W, HT Y, X L, SL Y, FJ Y, B X, ZY J, S L, $K X Z, X C$ J, FC L, LQ W, JJ D, X T, SY L performed the experiments and contributed materials; $Q X Y$ and $S H$ $Y$ analyzed the data and wrote the paper;.

All authors have read and approved the manuscript.

Acknowledgments

The authors thank all the patients who participated in this study.

Registration number and name of trial register

ChiCTR-IPR-14005482; Chinese Clinical Trial Registry(ChiCTR】

\section{References}

1. Siegel R, Ma J, Zou Z, et al. Cancer statistics 2014. [J]. CA Cancer J Clin. 2014;64:9-29.

2. LIU Chang-xiao XIAO, Pei-gen PENG, Yong, et al.,Challenges in research and development of traditional Chinese medicines, Chinese Herbal Medicines, no. 1, pp. 1-28, 2009.

3. Ji Q, Luo Y, Wang W, et al. Research advances in traditional Chinese medicine syndromes in cancer patients. Journal of Integrative Medicine. 2016;14(1):12-21.

4. Qian C-L, Yan F, Song Y-Z, et al.Is the traditional Chinese medicine helpful for patients with hematologic malignant diseases? A meta-analysis of randomized controlled trials, World Journal ofMeta-Analysis, vol. 3, no.3, pp. 163-180, 2015.

5. Liu Z, Chen S, Cai J,et al., Traditional Chinese Medicine syndrome-related herbal prescriptions in treatment of malignant tumors, Journal of Traditional Chinese Medicine, vol. 33. 
6. no. 1, pp. 19-26, 2013.

7. Tian J, Wang W-H, Cao H-M, et al. Determination of matrine, sophoridine and oxymatrine in compound Kushen injection by HPLC. China Journal of Chinese Materia Medica. 2007;32(3):222-4.

8. Liang QI, Zhang J, Zhang Z. Determination of four alkaloids in compound Kushen Injection by high performance liquid chromatography with ionic liquid as mobile phase additive. Chin J Chromatogr. 2013;31(3):249-53.

9. Zhang Y, Hui F, Yang Y. et.al. Can Kushen injection combined with TACE improve therapeutic efficacy and safety in patients with advanced HCC? a systematic review and network metaanalysis.Oncotarget, 2017 Sep 15;8(63):107258-107272.

10. Wang W, You RL, Qin WJ, et al. Anti-tumor activities of active ingredients in Compound Kushen Injection[J]. Acta Pharmacol Sin. 2015;36:676-9.

11. Qian ZZ, Dan Y, Liu YZ, et al, Pharmacopoeia of the People's Republic of China (2010 edition): a milestone in development of China's healthcare, Chinese Herbal Medicines.

12. vol. 2, no. 2, pp. 157-160, 2010.

13. Sun M, Cao H, Sun L,et al. Antitumor activities of kushen:literature review,Evidence-Based Complementary and Alternative Medicine. vol. 2012Article ID. 2012;373219:11 pages.

14. Ma X, Li R-S, Wang J, et al., The therapeutic efficacy and safety of compound kushen injection combined with transarterial chemoembolization in unresectable hepatocellular carcinoma: an update systematic review and meta-analysis, Frontiers in Pharmacology, vol. 7, article 70, 2016.

15. Xu W, Lin H, Zhang Y, et al., Compound Kushen Injection suppresses human breast cancer stem-like cells by downregulating the canonical Wnt/-catenin pathway, Journal of Experimental and Clinical Cancer Research, vol. 30, no. 1, article 103, 2011.

16. Yin L, Zheng WL, Sun QZ, et al. Meta-analysis of efficiency in treatment of gastric cancer by compound Kushen injection combined with chemotherapy,Chinese. Journal of Experimental Traditional Medical Formulae. 2013;19(14):325-30.

17. Wang ZY, Li GS, Huang HX. Clinical observation on treatment of 75 mid-late stage cancer patients with Yanshu injection. Chinese Journal of Integrated Traditional Western Medicine. 2006;26(8):6814.

18. Chen J, Mei Q, Xu Y-C, et al. Effects of Matrine injection on T-lymphocyte subsets of patients with malignant tumor after gamma knife radiosurgery. Journal of Chinese Integrative Medicine. 2006;4(1):78-9.

19. Yanju B, Yang L, Hua B, et al., A systematic review and meta analysis on the use of traditional Chinese medicine compound kushen injection for bone cancer pain, Supportive Care in Cancer, vol. 22, no. 3, pp. 825-836, 2014.

20. Aaronson NK, Ahmedzai S, Bergman B, et al. The European Organisation for Research and Treatment of Cancer QLQ-C30: a quality-of-life instrument for use in international clinical trials in oncology INCI 1993; 85: 365-376. 
21. https://ctep.cancer.gov/protocolDevelopment/electronic_applications/ctc.htm\#ctc_40, Common Terminology Criteria for Adverse Events v4.0 (CTCAE v4.03), June 14, 2010.

22. Guangxin R, Hegen $\mathrm{LI}$, Zhao L, et al. Effect of method of strengthening genuine qi on tumor microenvironment of advanced non-small cell lung cancer. Journal of Liaoning University of Traditional Chinese Medicine. 2013;no. 6:157-9.

23. Zhao Zhongquan L, Hehe Ju, Ying. Effect of compound Kushen injection on T-cell subgroups and natural killer cells in patients with locally advanced non-small-cell lung cancer treated with concomitant radiochemotherapy, J Tradit Chin Med. 2016 February 15; 36(1): 14-18.

24. Zhao Z, Fan H, Higgins T, et al. Fufang Kushen injection inhibits sarcoma growth and tumorinduced hyperalgesia via TRPV1 signaling pathways[J]. Cancer letters. 2014;355:232-41.

25. Xu W, Lin H, Zhang Y, et al. Compound Kushen Injection suppresses human breast cancer stem-like cells by down-regulating the canonical Wnt/ $\beta$-catenin pathway [J]. J Exp Clin Cancer Res. 2011;30:103.

26. Tian J, Wang WH, Gao HM, et al. Determination of matrine, sophoridine and oxymatrine in Compound Kushen Injection by HPLC. Zhongguo Zhong Yao Za Zhi. 2007;32:222-4.

27. Feng YL, Pan SJ, Yang NJ, et al. Meta-analysis on treatment of advanced non-small cell lung cancer with compound matrine injection in combination with Gemcitabine and Cisplatin chemotherapy. Mod J Integr Tradit Chin West Med. 2015;24:2303-6.

28. Lu L, Yang H, Teng ZY. Systematic review on compound sophoraflavescens injection adjuvant chemotherapy in the treatment of lung cancer. China Pharm. 2011;24:2267-70.

29. Wagner H. Image-guided conformal radiation therapy planning and delivery for non-small-cell lung cancer. Cancer control: journal of the Moffitt Cancer Center. 2003;10:277-88.

30. Qin JN. Compound kushen injection combined with chemotherapy for the treatment of elderly advanced non-small cell lung cancer. Contemp Med. 2014;09:132-3.

31. Lu L, Yang H, Teng ZY. Systematic review on compound sophoraflavescens injection adjuvant chemotherapy in the treatment of lung cancer. China Pharm. 2011;24:2267-70.

\section{Tables}

Due to technical limitations, the tables are only available as a download in the supplemental files section.

\section{Supplementary Files}

This is a list of supplementary files associated with this preprint. Click to download.

- Tables.pdf

- CONSORT2010Checklist.doc

- CONSORTExtensionforAbstractsChecklist.doc 